\section{Práticas e mudanças no comportamento alimentar na população de Brasília, Distrito Federal, Brasil}

\author{
Behavioral changes in food intake practices \\ in the population of Brasilia, Federal \\ District, Brazil
}

Maria Cristina Sebba Marinho 1

Edgar Merchán Hamann 2

Ana Carolina da Cunha Floresta Lima 3

\author{
1,2 Departamento de Saúde Coletiva. Faculdade de Ciências da \\ Saúde. Universidade de Brasília. Campus Universitário Darcy \\ Ribeiro.Asa Norte. Brasília, DF,Brasil. \\ CEP: 70.910-900. E-mail: csebba@unb.br \\ 3 Departamento de Nutrição. Faculdade de Ciências da Saúde. \\ Universidade de Brasília, DF,Brasil.
}

\begin{abstract}
Objectives: to investigate food habits changes in the population of Brasília, Federal District through variables such as schooling, income and sex.

Methods: A cross sectional study, with a probabilistic sample of 500 homes through interviews of people aged over 15. The interviews were held at the Plano Piloto, Cidades Satélites and Vila Estrutural regions of the Federal District between March 2003 and March 2004. The sample landmark was defined based on Instituto Brasileiro de Geografia $e$ Estatística census sectors related to the 2000 demographic census. Twenty census sectors were randomly selected among the 2548 in the Federal District.

Results: daily food intake consisted of: rice, beans, bread, fruit and vegetables. Chicken weekly consumption (85.0\%), beef (76.0\%), pasta (69.6\%) and eggs (62.0\%). The majority (71.0\%) reported concern with healthy nourishment. Only $37 \%$ of the respondents reported food habits change in the last six months and among them $32.6 \%$ change was related to the wish of losing weight, $25.4 \%$ to health concerns and $20.0 \%$ to food reeducation. Change was maintained by $84.2 \%$ of the respondents.

Conclusions: data indicate concern with healthy nourishment and change in food habits. Reasons reported for change are related to aesthetics and health concerns. Income and schooling are significant predictors of changes in food habits.
\end{abstract}

Key words Food habits, Behavioral change, Food survey

\section{Resumo}

Objetivos: investigar as práticas e mudanças alimentares na população de Brasília, Distrito Federal, com as variáveis escolaridade, renda e sexo.

Métodos: estudo transversal, com uma amostra probabilística de 500 domicílios, com entrevista a pessoas $>15$ anos. As entrevistas foram feitas no Plano Piloto, Cidades Satélites e Vila Estrutural do Distrito Federal, entre março de 2003 e março de 2004. O marco amostral foi definido com base nos setores censitários do Instituto Brasileiro de Geografia e Estatística, referentes ao censo demográfico de 2000. Foram sorteados 20 setores censitários dentre os 2548 existentes no Distrito Federal.

Resultados: os alimentos consumidos diariamente foram: arroz, feijão, pão, frutas, legumes e verduras. Consumo semanal de frango (85,0\%), carne bovina (76,0\%), massas $(69,6 \%)$ e ovos $(62,0 \%)$. A maioria $(71,0 \%)$ relatou preocupar-se com alimentação saudável. Apenas, $37 \%$ dos entrevistados relataram mudança alimentar nos últimos seis meses, e dentre eles 32,6\% atribuíram à perda de peso, 25,4\% à preocupação com a saúde e 20,0\% à reeducação alimentar. A mudança foi mantida por $84,2 \%$ dos respondentes.

Conclusões: os dados apontam a preocupação com a alimentação saudável e mudanças nas práticas alimentares. As razões alegadas para as mudanças estão relacionadas com a estética e preocupação com a saúde. Renda e escolaridade constituem preditores significativos das mudanças nas práticas alimentares.

Palavras-chave Práticas alimentares, Mudança comportamental, Inquérito alimentar 


\section{Introdução}

Práticas alimentares têm repercussões importantes no estado da saúde. Sabe-se que a promoção de mudanças nas práticas alimentares faz parte das metas para atingir a saúde. São necessárias mudanças baseadas na escolha e no preparo dos alimentos de maneira que promovam a adoção de uma alimentação mais saudável.

O Brasil, nas últimas décadas, passou por uma transição nutricional na qual os padrões alimentares mais tradicionais, como cereais, raízes e tubérculos foram sendo progressivamente substituídos por uma alimentação mais ocidental, isto é, consumo de alimentos mais ricos em gorduras e açúcares, a qual, aliada à diminuição progressiva de exercício físico, converge para o aumento no número de casos de sobrepeso e obesidade. Essas mudanças nos padrões de consumo têm colocado a população brasileira em maior risco de doenças crônicas. 1,2

É sabido que determinadas práticas alimentares constituem fatores protetores de condições mórbidas como as doenças cardiovasculares, diabetes, vários tipos de neoplasias e a obesidade, entre outras. Fornecer subsídios para a implementação de intervenções que promovam mudanças de práticas alimentares é o propósito de inúmeros estudos científicos.

No Brasil, desde os primeiros relatos de nossa história, há referências aos hábitos alimentares das populações. É notável a importância atribuída por Gilberto Freire e Luís da Câmara Cascudo aos fatores sócio-culturais e econômicos relacionados à alimentação brasileira. ${ }^{3,4}$ Entretanto, essas referências não se transformaram em pesquisas que pudessem revelar, de forma mais sistemática, as práticas alimentares dos diferentes segmentos da população brasileira.

Os poucos estudos de base populacional realizados no Brasil têm focalizado problemas nutricionais da dieta. Podem ser citados, como exemplos, o Estudo Nacional sobre Despesa Familiar (ENDEF), realizado entre 1974 e 1975,5 a Pesquisa Nacional sobre Saúde e Nutrição (PNSN), realizada em 1989,6 a pesquisa Condição Nutricional da População Brasileira: Adultos e $\operatorname{Idosos}^{7}$ e, mais recentemente, a Pesquisa sobre Padrões de Vida, realizada entre 1996 e 1997. ${ }^{8}$ Esses estudos foram de grande importância porque permitiram avaliar a magnitude dos agravos nutricionais mais relevantes. Segundo Sichieri et al., ${ }^{9}$ em geral, a maioria dos trabalhos de avaliação do consumo alimentar de populações baseia-se no cômputo dos macronutrientes no respectivo consumo calórico e no consumo dos micronutrientes associados mais freqüentemente ou às deficiências ou às doenças crônicas não transmissíveis da referida população.

A World Health Organization (WHO), $10 \mathrm{em}$ 1998, sugeriu que as avaliações alimentares deveriam basear-se em consumo de alimentos e não em nutrientes da dieta. Mais recentemente, no Brasil, destacam-se publicações focalizando os alimentos, isto é, pesquisas relativas ao padrão de consumo alimentar da população. ${ }^{11}$ Essas pesquisas deram origem a vários tipos de estudos de avaliação de padrões de consumo alimentar. ${ }^{12}$ Sichieri13 avaliou os fatores que explicam diferentes padrões de consumo alimentar da população do município do Rio de Janeiro. Garcia ${ }^{14}$ avaliou as práticas alimentares no meio urbano, no centro da cidade de São Paulo. O objetivo principal de suas pesquisas foi explorar a dimensão da comida no modo de vida urbano, tendo em vista as implicações que esse modo de vida tem nas práticas alimentares.

À medida que os estudos epidemiológicos passaram a demonstrar associação entre a dieta e algumas doenças não transmissíveis, a sociedade vem a se preocupando em realizar mudanças nos padrões alimentares. Poucos estudos têm demonstrado a freqüência da mudança comportamental em práticas alimentares. 15 Tais mudanças podem ser os resultados de políticas públicas e, eventualmente, de práticas terapêuticas.

Estudos epidemiológicos têm sido realizados com o objetivo de investigar possíveis relações entre alimentos e nutrientes das dietas. Para que essas associações possam ser bem investigadas é fundamental analisar a dieta pregressa ou habitual, que caracteriza o consumo alimentar durante um longo período. 16

No ambiente familiar, são geradas práticas que expressam valores, crenças e aspirações que são vividas no cotidiano como parte de um hábito importante na conformação dos indivíduos. Na idade infantil, cabe aos pais preparar a dieta dos filhos, mas quando a criança passa a freqüentar a escola e a conviver com outras crianças, ela conhecerá hábitos, alimentos e preparações diferentes, que podem ser ou não saudáveis. E já que são os adultos que escolhem os alimentos para as crianças, os pais, naturalmente, são os primeiros responsáveis para a transmissão de um padrão alimentar saudável, isto é, uma alimentação moderada, variável e equilibrada. ${ }^{17}$

Aquino e Philippi 18 realizaram um estudo no município de São Paulo com 718 crianças de até cinco anos de idade, e verificaram que, dentre os alimentos industrializados mais consumidos, encon- 
trou-se o açúcar com $(66,3 \%)$ e biscoitos $(40 \%)$. Os dados sugerem que houve um consumo rico em calorias, em vez de nutrientes. Os jovens mostram-se mais susceptíveis ao consumo inadequado desses alimentos. Em um estudo feito com adolescentes, de 10 a 18 anos de idade, foram ana-lisados os alimentos mais consumidos e verificou-se o excesso de consumo de balas, doces, chocolates, chicletes, salgadinhos, pastéis, coxinhas, etc. 19 Nesse estudo, pôde-se observar uma carência de consumo de frutas, verduras e legumes. Sabe-se que é comum, entre os adolescentes, substituir almoço e o jantar por lanches com guloseimas, não comer em horário certo e seguir rituais grupais de não consumir determinados alimentos. Assim, o uso freqüente de alimentos de fácil consumo, ou o fato de "pular" refeições, aumenta, sobremaneira, o risco nutricional dos adolescentes.

Crianças e adolescentes são influenciados pelas imagens de jovens modelos de moda extremamente magras e por relatos de que o indivíduo que atingir esse ideal de beleza passa a ter sucesso nos relacionamentos sociais, e de que o indivíduo gordo é socialmente inaceitável. 20 Waterhouse 21 verificou que $50 \%$ de meninas com nove anos e $80 \%$ de dez anos de idade faziam dietas com receio de engordar.

O objetivo do presente estudo consistiu em investigar os padrões de consumo alimentar da população do Distrito Federal, mediante uma abordagem quantitativa. Pretendeu-se também quantificar aspectos associados à mudança comportamental nas práticas alimentares, analisando suas relações com variáveis socioeconômicas e demográficas.

\section{Métodos}

Trata-se de um estudo epidemiológico transversal, no qual foram obtidos dados de prevalência de relatos de práticas e de mudanças no comportamento alimentar, bem como de variáveis demográficas e socioeconômicas. A pesquisa foi realizada no Departamento de Saúde Coletiva da Universidade de Brasília (UnB) localizada em Brasília, Distrito Federal, Brasil. Foi analisada a mudança alimentar, nos últimos seis meses, entre as varáveis, renda, sexo, idade, e escolaridade.

A coleta de dados foi realizada com uma amostra de 500 respondentes, com indivíduos maiores de 15 anos. O tamanho da amostra foi calculado para detectar diferenças ou proporções de $13,5 \%$ na adequação de consumo de legumes segundo nível de renda, de acordo com prevalências observadas em outros estudos. Foi calculado com um erro $\alpha$ menor de $5 \%$ e poder $(1-\beta)=80 \%$. O marco amostral, no Distrito Federal, foi definido com base nos setores censitários da Fundação Instituto Brasileiro de Geografia e Estatística (FIBGE), referentes ao censo demográfico de 2000. Foram sorteados 20 setores censitários dentre os 2548 existentes no Distrito Federal, a partir de um programa de escolha aleatória: Epi-info 6.0. As localidades dos setores sorteados foram: o Plano Piloto (Asa Sul, Asa Norte, Sudoeste), Cidades Satélites (Águas Claras, Ceilândia, Gama, Guará, Sobradinho, e Planaltina) e a Vila Estrutural.

O primeiro passo para a escolha dos domicílios, foi o reconhecimento da área para verificar o número de domicílios daquele setor. Em seguida, calculou-se a fração amostral, tendo por base o número de domicílios dividido por 25 que corresponde ao número de questionários aplicados em cada setor. Para a seleção sistemática dos domicílios, tomando como exemplo, uma fração amostral igual a cinco, sorteou-se um dos cinco primeiros domicílios do setor, e a partir desse, era selecionado o quinto domicílio posterior, e assim sucessivamente, até um total de 25 domicílios por setor. Os domicílios foram substituídos quando o morador presente recusava-se a participar da pesquisa, ou quando não havia moradores. Nessa situação, o entrevistador se dirigia ao domicílio de número superior ao sorteado.

Nos domicílios selecionados, fez-se sorteio de um morador maior de 15 anos usando fichas com as denominações: pai, mãe, filhos e agregados, conforme o grau de parentesco com o chefe da família e com a idade. Os filhos e agregados foram ordenados seguindo uma ordem decrescente de idade. Caso o morador sorteado não aceitasse participar da pesquisa, fazia-se um novo sorteio dentre os moradores do mesmo domicílio. Não encontrando em casa o morador sorteado, perguntava sobre o melhor horário para o retorno e realização da entrevista. Retornava-se no horário marcado e, se o sorteado não estivesse, retornava-se de uma a três vezes neste mesmo domicílio; ainda, se não fosse possível a realização da entrevista, recorria-se ao local de trabalho (raras exceções). Em último caso, fazia-se um novo sorteio entre os que estivessem presentes no domicílio. O tempo médio de duração da entrevista foi de 25 minutos.

A coleta de dados foi realizada de março de 2003 a março de 2004, mediante a aplicação de um questionário estruturado e pré-testado com 65 questões, por estudantes da Faculdade de Saúde, devidamente treinados e identificados como participantes de projeto de pesquisa da Universidade de Brasília. O período de um ano de aplicação dos questionários foi 
devido a resistência dos moradores de domicílios selecionados e ao procedimento da re-aplicação conforme explicado acima, atrasando, com isso, o cronograma original. Os intervalos de classe utilizados no questionário para as diferentes categorias de renda foram dez, de "sem rendimento" até "mais de R \$ 6.000,00". Neste artigo foram utilizados, para efeito de análise, apenas dois intervalos. Observa-se que a renda média familiar no Distrito Federal é maior que nos demais estados, assim, a maior concentração da renda se deu no intervalo acima de $\mathrm{R} \$ 960,00$.

Foi questionada a preferência alimentar, segundo outras características, tais como: a forma de consumir alimentos (assados, fritos, cozidos); uso de sal de adição aos alimentos já preparados; o que se usa para adoçar os alimentos (açúcar, adoçante ou natural); sobre a preferência de alimentos gordurosos ou não; se costumam trocar jantar por lanches; se fizeram alguma mudança alimentar nos últimos seis meses, e se ela foi mantida; se evitam algum tipo de alimento e as razões aduzidas. O questionário continha outras variáveis não exploradas no presente artigo devido ao número limitado de tabelas, como, por exemplo, forma de aquisição, preparo de alimentos, conceito de alimentação adequada, prática de exercícios físicos, entre outras.

Foi determinada a razão de prevalências entre variáveis socioeconômicas e demográficas e mudança comportamental. Os fatores associados à mudança comportamental foram avaliados mediante a aplicação do teste qui-quadrado para as categorias de sexo, idade, escolaridade e renda. O nível de significância considerado foi $5 \%$.

A regressão logística foi realizada mediante o método backward conditional, definida como variável dependente a mudança de hábitos de consumo nos último seis meses. Posteriormente, foram incluídas no modelo as variáveis independentes mais relevantes como renda familiar, idade, sexo, escolaridade, local de moradia e preocupação com a saúde. Os dados foram digitados e processados no programa Excel e as análises estatísticas pelo programa, SPSS for Windows versão 10.0 .

Foram cumpridos todos os requisitos para pesquisa com seres humanos, previstos pelo Parecer de Aprovação da Comissão de Ética, conforme resolução do Conselho Nacional de Saúde, sob guarda do Departamento de Saúde Coletiva da Faculdade de Ciências da Saúde da Universidade de Brasília.

\section{Resultados}

Foram analisados 500 questionários, não havendo perdas. Predominou o sexo feminino. A idade da população estudada variou de 15 a 89 anos, com média de 35 e mediana de 31 anos. Em relação à escolaridade, os dados mostraram um alto índice de segundo grau completo e de nível superior. Quanto à renda familiar, $41,4 \%$ dos informantes ganhavam até quatro salários mínimos ( $\mathrm{R} \$ 959,00)$, ressaltando-se que apenas $2,4 \%$ do total declararam não ter renda. A renda familiar média foi de aproximadamente $\mathrm{R} \$ 2.194,00$ e a mediana, foi $\mathrm{R} \$ 1.594,00$.

Em relação ao local de moradia, 75,0\% dos entrevistados moram nas Cidades Satélites. Incluem-se, nesse percentual, 10,0\% dos entrevistados, que residem numa área de ocupação irregular (Vila Estrutural).

A amostra $(n=500)$ caracterizou-se, como relatado na Tabela 1 , por predominância do sexo feminino, sendo a maioria de 25 anos ou mais, com escolaridade de segundo grau completo e de nível superior. A renda familiar total dos respondentes, levando em consideração o salário mínimo da época (1 SM=240) era até $3 \mathrm{SM}$.

A Tabela 2 apresenta a freqüência de consumo de alimentos, como o arroz $(79,4 \%)$, o feijão $(71,2 \%)$ e o pão $(56,0 \%)$, que apresentaram as maiores freqüências de consumo diário. Além desses alimentos, foi observado um alto consumo semanal de frango $(85,0 \%)$, carne bovina $(76,1 \%)$, massas $(69,6 \%)$ e ovos $(62,7 \%)$, assim como a ingestão freqüente de frutas, verduras e legumes que são consumidos diariamente por cerca da metade dos entrevistados. Dentre os alimentos de menor consumo estão a carne suína, rejeitada por $81,2 \%$, e o peixe, por $66,4 \%$ dos respondentes. A maioria dos informantes relatou não consumir carne suína por ser um tipo de carne, ainda não confiável e por ser uma carne gordurosa e de difícil acesso. Quanto ao peixe, a maioria relatou o alto preço e a dificuldade de acesso.

A Tabela 3 apresenta a prevalência de relato sobre algumas práticas que indicam preocupação com a saúde. Destaca-se que a preferência de carne vermelha consumida sem a gordura aparente, equivale a $45,5 \%$, o consumo de carne de frango, sem a pele, cor-responde a $47,0 \%$. Tais percentuais foram um pouco mais baixos para o consumo de carnes sem nervos e pele $(35,0 \%)$. Quanto à preferência na forma de preparação das carnes, vermelha e de frango, a opção principal foi pela carne cozida (cerca de $50,0 \%$ ). Quando questionados pela razão na forma de preparo de carnes, 63,0\% atribuíram a escolha ao sabor e $26,2 \%$ à preocupação com a saúde.

Em relação à atitude com respeito à ingestão de gorduras, predominou a tendência de consumir 
Características sócio-demográficas da população de estudo. Brasília, Distrito Federal, 2003 a 2004.

\begin{tabular}{|c|c|c|c|c|}
\hline Variável & Classes & $\mathbf{N}$ & $\%$ & IC95\% \\
\hline \multirow[t]{2}{*}{ Sexo } & Masculino & 178 & 35,6 & $31,4-39,8$ \\
\hline & Feminino & 322 & 64,4 & $60,2-68,6$ \\
\hline \multirow[t]{4}{*}{ Idade } & $15-30$ & 234 & 46,9 & $42,5-51,3$ \\
\hline & $31-45$ & 158 & 31,7 & $27,6-35,8$ \\
\hline & $46-60$ & 59 & 11,8 & $9,0-14,6$ \\
\hline & Acima de 60 & 48 & 9,6 & $7,0-12,2$ \\
\hline \multirow[t]{5}{*}{ Escolaridade } & Não estudou & 15 & 3,0 & $1,5-4,5$ \\
\hline & $1^{\circ} \mathrm{Grau}$ & 180 & 36,0 & $31,8-40,2$ \\
\hline & $2^{\circ} \mathrm{Grau}$ & 176 & 35,2 & $31,0-39,4$ \\
\hline & Superior & 124 & 24,8 & $21,0-28,6$ \\
\hline & Sem informação & 5 & 1,0 & $0,1-1,9$ \\
\hline \multirow[t]{5}{*}{ Renda familiar } & Até $\mathrm{R} \$ 959,00$ & 195 & 39,0 & $34,7-43,3$ \\
\hline & $\mathrm{R} \$ 960,00$ a $\mathrm{R} \$ 2.879,00$ & 117 & 23,4 & $19,7-27,1$ \\
\hline & Acima de $R \$ 2.880,00$ & 132 & 26,4 & $22,5-30,3$ \\
\hline & Sem rendimento & 12 & 2,4 & $1,1-3,7$ \\
\hline & Não informou & 44 & 8,8 & $6,3-11,3$ \\
\hline \multirow[t]{2}{*}{ Local de moradia } & Plano Piloto & 125 & 25,0 & $21,2-28,8$ \\
\hline & Cidades Satélites & 375 & 75,0 & $71,2-78,8$ \\
\hline
\end{tabular}

\section{Tabela 2}

Freqüência de consumo de determinados alimentos em indivíduos menores de 15 anos. Brasília, Distrito Federal, 2003 a 2004.

\begin{tabular}{|c|c|c|c|c|c|c|}
\hline Alimentos & Não consome (\%) & IC95\% & Consumo semanal (\%) & IC95\% & Consumo diário (\%) & IC95\% \\
\hline Arroz & 1,4 & $0,4-2,4$ & 19,2 & $15,7-22,7$ & 79,4 & $75,9-82,9$ \\
\hline Pão & 10,4 & $7,7-13,1$ & 33,6 & $29,5-37,7$ & 56,0 & $51,6-60,4$ \\
\hline Massas & 24,0 & $20,3-27,7$ & 69,6 & $65,6-73,6$ & 6,4 & $4,3-8,5$ \\
\hline Feijão & 5,0 & $3,1-6,6$ & 23,8 & $20,1-27,5$ & 71,2 & $67,2-75,2$ \\
\hline Frango & 8,6 & $6,1-11,1$ & 85,0 & $81,9-88,1$ & 6,4 & $4,3-8,5$ \\
\hline Carne bovina & 12,2 & $9,3-15,1$ & 76,1 & $72,4-79,8$ & 11,6 & $8,8-14,4$ \\
\hline Carne suína & 81,2 & $77,8-84,6$ & 18,4 & $15,0-21,8$ & 0,4 & $-0,2-1,0$ \\
\hline Peixe & 66,4 & $62,3-70,5$ & 32,2 & $28,1-36,6$ & 1,4 & $0,4-2,4$ \\
\hline Ovo & 32,7 & $28,6-36,8$ & 62,1 & $57,8-66,4$ & 5,3 & $3,3-7,3$ \\
\hline Frutas & 9,2 & $6,7-11,7$ & 41,5 & $37,2-45,8$ & 49,3 & $44,9-53,7$ \\
\hline Verduras & 10,4 & $7,7-13,1$ & 40,0 & $35,7-44,3$ & 49,6 & $45,2-54,0$ \\
\hline Legumes & 9,2 & $6,7-11,7$ & 45,4 & $41,0-49,8$ & 45,4 & $41,0-49,8$ \\
\hline Doces & 40,9 & $36,6-45,2$ & 43,9 & $39,6-48,2$ & 15,2 & $12,1-18,3$ \\
\hline
\end{tabular}


Características das práticas alimentares na população. Brasília, Distrito Federal, 2003 a 2004.

\begin{tabular}{|c|c|c|c|c|}
\hline Variável & Classes & $\mathbf{N}$ & $\%$ & IC95\% \\
\hline O que se retira da & Gordura visível & 227 & 45,5 & $41,1-49,9$ \\
\hline carne vermelha & Nervos & 32 & 6,4 & $4,3-8,5$ \\
\hline \multirow[t]{3}{*}{ quando consumida } & Gordura e nervos & 140 & 28,1 & $24,2-32,0$ \\
\hline & Nada & 80 & 16,0 & $12,8-19,2$ \\
\hline & Não consome carne vermelha & 20 & 4,0 & $2,3-5,7$ \\
\hline O que se retira do & Gordura visível & 17 & 3,4 & $1,8-5,0$ \\
\hline frango quando & Nervos & 5 & 1,0 & $0,1-1,9$ \\
\hline \multirow[t]{4}{*}{ consumido } & Pele & 236 & 47,4 & $43,0-51,8$ \\
\hline & Gordura, nervos e pele & 176 & 35,3 & $31,1-39,5$ \\
\hline & Nada & 59 & 11,8 & $9,0-14,6$ \\
\hline & Não consome frango & 5 & 1,0 & $0,1-1,9$ \\
\hline Preferência de & Assadas & 99 & 20,0 & $16,5-23,5$ \\
\hline forma de preparo & Cozidas & 242 & 49,0 & $44,6-53,4$ \\
\hline \multirow[t]{2}{*}{ de carnes } & Fritas & 103 & 20,9 & $17,3-24,5$ \\
\hline & Grelhadas & 50 & 10,1 & $7,5-12,7$ \\
\hline Motivo para & Sabor & 309 & 62,8 & $58,6-67,0$ \\
\hline preferência do & Rendimento & 19 & 3,9 & $2,2-5,6$ \\
\hline \multirow[t]{4}{*}{ preparo das carnes } & Aparência & 9 & 1,8 & $0,6-3,0$ \\
\hline & Preocupação com saúde & 129 & 26,2 & $22,3-30,1$ \\
\hline & Outros motivos & 21 & 4,3 & $2,5-6,1$ \\
\hline & Não consome carne & 5 & 1,0 & $0,1-1,9$ \\
\hline Atitudes de & Com muita gordura & 39 & 7,8 & $5,4-10,2$ \\
\hline consumo de & Com pouca gordura & 365 & 73,3 & $69,4-77,2$ \\
\hline \multirow[t]{2}{*}{ gordura } & Sem gordura & 28 & 5,6 & $3,6-7,6$ \\
\hline & Consome o que vem no prato & 66 & 13,3 & $10,3-16,3$ \\
\hline Tipo de leite & Leite de soja & 12 & 2,4 & $1,1-3,7$ \\
\hline \multirow[t]{4}{*}{ consumido } & Leite integral & 294 & 59,0 & $54,7-63,3$ \\
\hline & Leite Semidesnatado & 16 & 3,2 & $1,7-4,7$ \\
\hline & Leite desnatado & 85 & 17,1 & $13,8-20,4$ \\
\hline & Não costuma consumir leite & 91 & 18,3 & $14,9-21,7$ \\
\hline Motivo de evitar & Religioso & 2 & 0,6 & $-0,1-1,3$ \\
\hline \multirow[t]{4}{*}{ algum alimento } & Estética corporal & 20 & 6,3 & $4,2-8,4$ \\
\hline & Saúde (prevenção) & 95 & 30,2 & $26,2-34,2$ \\
\hline & Saúde (tratamento) & 41 & 13,0 & $10,1-15,9$ \\
\hline & Não gosta & 157 & 49,8 & $45,4-54,2$ \\
\hline Costuma trocar & Sim & 246 & 49,4 & $45,0-53,8$ \\
\hline \multirow[t]{2}{*}{ jantar por lanche } & Não & 151 & 30,3 & $26,3-34,3$ \\
\hline & Às vezes & 101 & 20,3 & $16,8-23,8$ \\
\hline
\end{tabular}


alimentos com pouca gordura $(73,3 \%)$. Por outro lado, é oportuno relatar que a maioria dos entrevistados preferiu o leite integral $(59,0 \%)$. Os que não costumvam tomar leite representavam $18,0 \%$.

Dentre os entrevistados que evitam algum alimento, a razão mais alegada para o não consumo era por não gostar. Já os que evitavam alimentos por razões profiláticas ou terapêuticas equivaliam a $43 \%$. Outro fator relevante dos respondentes é quanto ao hábito de substituir o jantar por lanches.

A Tabela 4 apresenta a freqüência de mudanças alimentares recentes e alguns fatores correlatos. Dentre os entrevistados, 186 (37\%) responderam que fizeram mudança alimentar nos últimos seis meses, e para esses perguntou-se qual o motivo que levou à mudança, com opção de até duas respostas (num total de 248 respostas). Em relação a esses motivos, $32,6 \%$ relataram a perda de peso, seguida da preocupação com a saúde $(25,4 \%)$ e reeducação alimentar (20,6\%).

Em relação aos 186 respondentes que fizeram mudança alimentar, 84,2\% mantiveram a mudança. Dentre os respondentes, os que disseram conhecer outra pessoa que tenha mudado seus hábitos alimentares, nos últimos seis meses, equivalem a $41,2 \%$. Destaca-se que $71 \%$ da amostra total se preocupava em ter uma alimentação adequada, o que indica uma preocupação com a saúde.

A Tabela 5 apresenta a análise de associação entre as características sócio-demográficas e a mudança alimentar nos últimos seis meses. A mudança alimentar revelou um pequeno predomínio em mulheres, nas faixas mais jovens (15 e 24 anos), e nas pessoas que se classificavam como sendo da raça negra. Porém, nenhuma dessas associações foi significante. Por outro lado, os fatores sobre renda familiar, escolaridade e local de moradia mostraram-se significativamente associados à mudança alimentar. Os setores de nível socioeconômico mais elevado foram os que adotaram esse comportamento, com maior freqüência.

Na regressão logística, das seis variáveis inde-

Tabela 4

Mudança dos hábitos alimentares nos últimos seis meses na população. Brasília, Distrito Federal, 2003 a 2004.

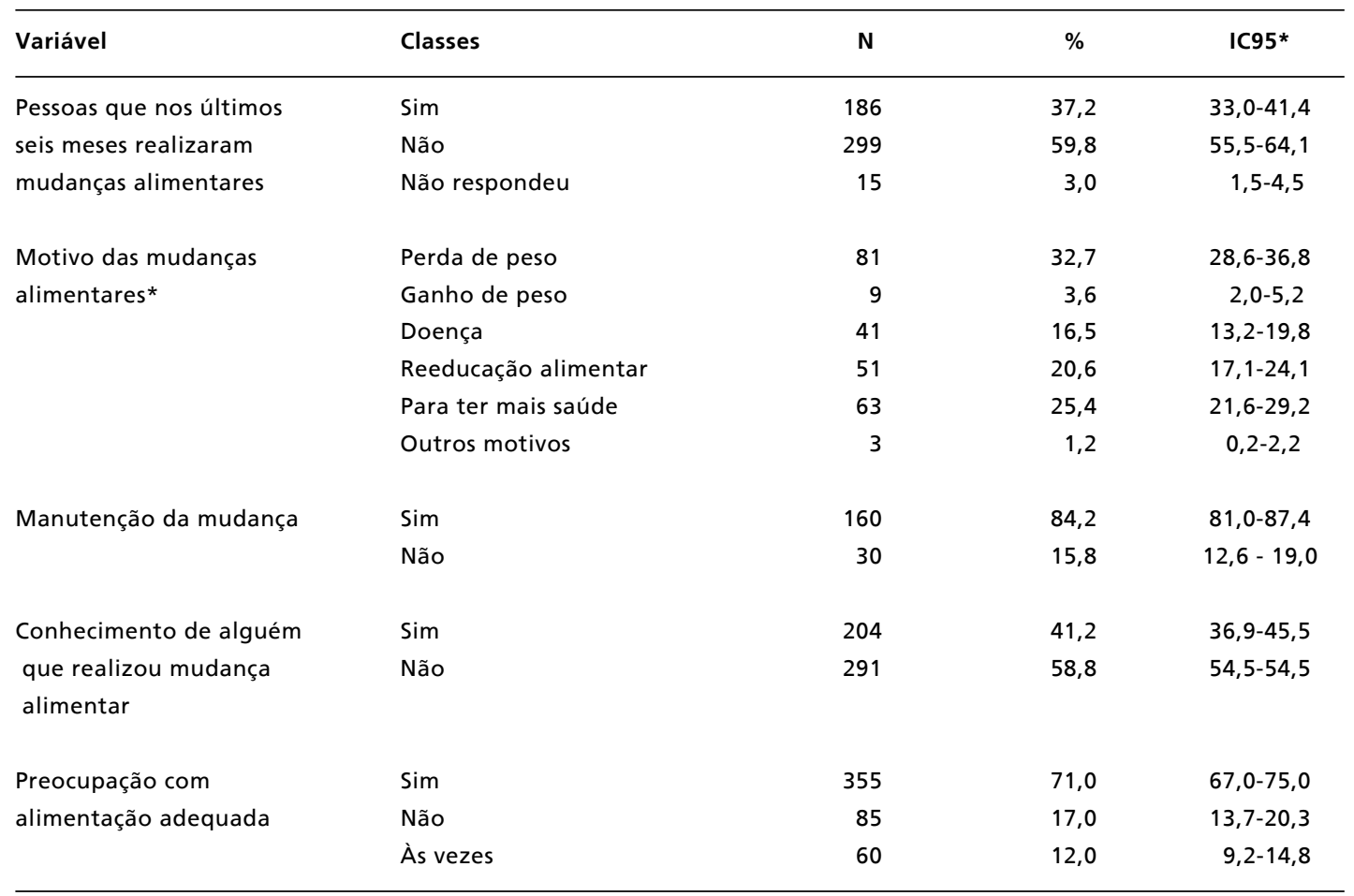

* Os entrevistados poderiam atribuir até dois motivos para as mudanças alimentares (248 respostas). 
Análise e associação das variáveis sócio-demográficas com a mudança alimentar. Brasília, Distrito Federal, 2003 a 2004

\begin{tabular}{|c|c|c|c|c|c|}
\hline Variável & Classes & $\begin{array}{l}\text { Prevalência de mudança } \\
\text { alimentar }\end{array}$ & $\begin{array}{c}\text { Razão de } \\
\text { prevalências }\end{array}$ & IC95\% & $\mathbf{p}^{*}$ \\
\hline \multirow[t]{2}{*}{ Sexo } & Feminino & 39,3 & \multirow{2}{*}{1,07} & \multirow{2}{*}{$0,84-1,36$} & \multirow{2}{*}{0,563} \\
\hline & Masculino & 36,6 & & & \\
\hline \multirow[t]{2}{*}{ Idade } & Até 24 anos & 42,9 & \multirow{2}{*}{1,18} & \multirow{2}{*}{$0,93-1,49$} & \multirow{2}{*}{0,171} \\
\hline & Acima de 24 anos & 36,4 & & & \\
\hline \multirow[t]{2}{*}{ Renda familiar } & Acima de $R \$ 960,00$ & 47,3 & \multirow{2}{*}{1,60} & \multirow{2}{*}{$1,240-2,06$} & \multirow{2}{*}{$<0,001$} \\
\hline & Até $\mathrm{R} \$ 959,00$ & 29,6 & & & \\
\hline \multirow[t]{2}{*}{ Escolaridade } & $2^{\circ} \mathrm{Grau}$ e Superior & 59,3 & \multirow{2}{*}{1,88} & \multirow{2}{*}{$1,52-2,33$} & \multirow{2}{*}{$<0,001$} \\
\hline & Não estudou e $1^{\circ} \mathrm{Grau}$ & 31,5 & & & \\
\hline
\end{tabular}

* Teste qui-quadrado.

pendentes, sexo, idade, grau de escolaridade, renda familiar, local de moradia e raça, somente duas permaneceram no modelo final. São elas, idade e grau de escolaridade. O cálculo dos logos, correspondentes a essas variáveis, revelou que pessoas de até 24 anos de idade e baixa escolaridade têm uma probabilidade de mudança alimentar de 0,38 , enquanto esse valor passa a ser 0,65 nos indivíduos da mesma faixa etária, porém, de maior escolaridade. As pessoas acima de 24 anos e baixa escolaridade têm uma probabilidade de 0,3 de fazer mudança alimentar, enquanto esse valor é de 0,56 entre indivíduos dessa faixa etária e com maior escolaridade.

As pessoas com maior probabilidade de fazer alguma mudança alimentar eram aquelas de idade menor ou igual a 24 anos e com escolaridade de segundo grau ou de nível superior. Pode-se constatar esse relato pelos coeficientes das variáveis no modelo de regressão logística (coef. Idade $=-0,393$ e coef. Escolaridade $=-1,115$ ).

\section{Discussão}

As práticas alimentares têm sido, tradicionalmente, objeto de pesquisas que procuram definir um perfil das opiniões e atitudes do cidadão brasileiro, residente nas grandes cidades, a respeito de alimentação. A cidade de Brasília, DF, foi utilizada como base de pesquisa que procurou definir um perfil das opiniões e atitudes sobre alimentação.

Uma característica de Brasília é possuir uma população originária das diversas regiões do país. O processo de amostragem utilizado nesta pesquisa garante representatividade populacional para o Distrito Federal. Em estudos realizados para avaliar os padrões alimentares no Brasil, alguns autores mostram resultados nesse enfoque utilizando inquéritos alimentares como método.1,13,22,23

Dentre as limitações deste estudo é conveniente mencionar que, ao se tratar de um estudo transversal, torna-se difícil avaliar até que ponto os padrões de práticas alimentares estão incorporando mudanças induzidas por intervenções educativas ou de outra natureza. Essa limitação do estudo transversal pode ter sido incrementada pelo prolongado período de coleta de dados (um ano), em que co-intervenções poderiam mudar a realidade na fase final em relação ao começo do trabalho de campo. Da mesma forma, há dificuldade, em pesquisa domiciliar, em relação à delimitação da cronologia dos eventos.

Houve assim, a preocupação de contornar os vieses de memória mediante a delimitação de períodos de recordação recente. Outros tipos de pesquisa, por exemplo, estudos de coorte, esclareceriam tendências no consumo, mudanças de práticas alimentares associando-as a intervenções específicas. Dessa forma, não foi feito um aprofundamento sóciocultural conforme a recomendação de Garcia. ${ }^{23}$ Igualmente não se chegou a pesquisar as representações simbólicas que permeiam os comportamentos alimentares. Porém, foram incluídas as principais variáveis socioeconômicas e demográficas que demonstram ser preditoras da mudança comportamental. 
Foi verificada uma maior participação de indivíduos do sexo feminino, apesar dos cuidados metodológicos para garantir a amostra adequada da população. Mesmo fazendo novamente o sorteio dos moradores, quando não se encontravam em casa, voltando pela segunda ou terceira vez, ainda prevalecia o sexo feminino. A maior presença de mulheres no domicílio relaciona-se com seu predomínio em atividades domésticas, o que representa uma limitação das pesquisas domiciliares. Com a faixa etária ocorreu fato semelhante. Entre os entrevistados, a faixa etária de 15 a 30 anos foi a que teve maior freqüência, o que representou praticamente a metade da população. O censo de 2000 , também, mostrou a predominância dessa faixa de idade, que equivale a $33,3 \%$ da população.

Do ponto de vista histórico, Cascudo 4 já apontava a importância do arroz e do feijão como alimentos da tradição da comida brasileira. O grande destaque dado na mídia, em relação à alta freqüência do consumo de carboidratos, é corroborado nesta pesquisa em relação ao significativo consumo de arroz e pão. O alto consumo de carboidrato corrobora dados da Pesquisa de Orçamentos Familiares (POF), do IBGE, na qual se verifica o predomínio dos cereais, principalmente o arroz, como principal alimento brasileiro. ${ }^{2}$ Mattos e Martins 24 relataram, também, o consumo de alta freqüência de arroz e feijão, nas principais refeições, concluindo serem esses os itens básicos do padrão alimentar brasileiro.

Conforme os resultados do estudo multicêntrico, 25 que traçou um perfil do padrão alimentar, em cinco cidades brasileiras (Campinas, Curitiba, Goiânia, Ouro Preto e Rio de Janeiro), os 16 alimentos mais consumidos em ordem decrescente de prioridade em todas as faixas de renda apresentaram variação por nível salarial e entre os municípios estudados. Ao comparar os principais alimentos mais consumidos, houve variaçãos por nível de renda, como por exemplo, arroz, feijão, leite, óleo, pão francês, açúcar e macarrão, os quais foram os mais consumidos para renda de até meio salário mínimo per capita. Já para a faixa de renda mais alta, estes alimentos foram: pão de forma, pão francês, arroz, leite, feijão e açúcar. O estudo mostrou que o arroz e o feijão são consumidos em ambas as faixas de renda, embora com variação na ordem de salário. Enquanto arroz e o feijão ocuparam a primeira posição para os indivíduos de renda menor, para os indivíduos de renda menor, para os mais abastados, esses alimentos ocuparam o segundo lugar. De acordo com os resultados desse estudo, dentre as cidades pesquisadas, a de
Campinas foi a que teve o mais alto consumo de arroz e feijão, semelhante aos resultados encontrados para Brasília, na presente pesquisa.

A Pesquisa de Orçamento Familiar (POF), 26 de 1995 a 1996, apontou alguns resultados sobre modificações na alimentação brasileira: comparando com a POF de 1987 a 1988, foi demonstrada uma redução relativa no consumo de carnes de $17,13 \%$ para $12,9 \%$, legumes de $5,56 \%$ para $4,22 \%$, açúcares e derivados de $4,44 \%$ para $3,40 \%$. Os panificados (pão, biscoitos e outros) e os alimentos preparados ou semi-prontos aumentaram seu gasto no orçamento familiar. Apesar da redução com carnes, cresceu o consumo de frango (16,56\%) e houve um declínio no consumo de carboidratos como arroz, farinha de trigo, reduzido em 16,56\%. Outros alimentos que tiveram seu consumo reduzido foram feijão $(15,56 \%)$ e leite de vaca $(19,31 \%)$. Tais pesquisas endossam a premissa de que a alimentação tipicamente brasileira tem sofrido mudanças em direção à alimentação dos países desenvolvidos.

Esses resultados não corroboram os dados da presente pesquisa em relação ao consumo freqüente de arroz, pão e feijão. Contrariamente à POF, neste estudo, foi confirmado o consumo significativo de carboidratos (arroz e pão) e de feijão, suportando outros estudos.27 Constataram-se, ainda, as mudanças que ocorreram nos últimos seis meses prévios a aplicação do questionário, com a população acima ou igual a 25 anos. Os participantes passaram a comer menos sal, mais frutas, verduras, legumes e menos carne vermelha. Em dieta, recomendada por nutricionista ou médico, observouse uma preocupação tanto em adultos como em jovens abaixo de 25 anos. Quanto a dietas da moda, os mais adeptos são os jovens abaixo de 25 anos de idade.

O modo de vida urbano é o cenário dessas mudanças. As condições que ocorrem na vida cotidiana implicam na relação do indivíduo com seu meio, refletindo no modo de comer e de relacionarse com alimentação, induzindo alterações nas práticas alimentares. A pesquisa mostra que as pessoas que mudaram seus hábitos alimentares o fizeram em função da perda de peso, como principal motivo, evidenciando o valor que é dado à estética, sendo superior, inclusive, à preocupação com saúde. Entretanto, aspectos mais relacionados à saúde, também têm gerado mudanças alimentares.

A análise demonstrou que os respondentes com maior escolaridade foram os mais adeptos às mudanças, independente de outras variáveis estudadas.

Foi surpreendente o aparecimento da idade como 
uma variável relevante no modelo de regressão logística e, da mesma forma, que a categoria de implementação de mudança recente no comportamento alimentar, corresponda aos sujeitos mais jovens. Isso pode ser explicado por uma interação de alta escolaridade e de idade, sugerindo uma provável tendência recente que ultrapassa as mudanças de hábitos esperadas, ocorrendo mais habitualmente na faixa acima de 40 anos.

O consumo de frutas, verduras e legumes, como foi observado neste estudo, indica uma preocupação com a alimentação adequada e com a saúde. Isso reflete a relevância dada, atualmente, à importância do consumo desses alimentos para um adequado suprimento das necessidades de diversas vitaminas, minerais e de fibras, em razão das funções desses nutrientes na proteção contra doenças cardíacas, neoplásicas, diabetes e afecções gastrointestinais.

De acordo com relatos de estudos recentes, parece não haver dúvida de que a prevenção e controle de certas doenças estão relacionadas com estratégias de mudanças de hábitos alimentares. 15,24,28 A Pesquisa de Orçamento Familiar do Instituto Brasileiro de Geografia e Estatística 1995 a 1996, relata o declínio relativo do consumo dos alimentos in natura. $26 \mathrm{O}$ declínio atinge as classes de menor renda, o que provavelmente justifica as respostas que se referem aos preços e acesso como limitadores do consumo. A premência atual, por se prevenirem as moléstias derivadas da falta de consumo desses alimentos, representa o ponto central nos programas alimentares, o que tem sensibilizado as classes de maior renda. Neste estudo, foram justamente as classes de maior renda as que mudaram com maior frequiência os hábitos alimentares, nos seis meses prévios à entrevista.

A pesquisa levantou uma série de informações sobre as práticas alimentares no Distrito Federal e os resultados corroboram levantamentos anteriores quanto ao predomínio do chamado padrão brasileiro de alimentação. Entretanto, ressalta-se a maior mudança comportamental alimentar na população de maior escolaridade, que mostrou uma relação consistente com renda e local de moradia. As diferenças socioeconômicas constituem preditores mais significativos das mudanças nas práticas alimentares do que sexo, idade e raça. Deve-se destacar, no entanto, que a presente pesquisa não aborda o acesso aos alimentos de melhor qualidade nem as informações relacionadas com uma alimentação saudável; esses são fatores que também devem depender de condicionantes socioeconômicos. Tampouco, foram abordadas, neste estudo, as representações simbólicas que estão envolvidas tanto nas práticas quanto nas mudanças alimentares. Portanto, estudos complementares de cunho qualitativo sobre a cultura alimentar, também, devem contribuir à compreensão de aspectos subjetivos e culturais envolvidos nesses processos.

É oportuno ressaltar que informações sobre alimentação adequada são importantes porque, de imediato, mostram situações de risco, tanto de pessoas como de regiões, e permitem aos estados, municípios e ao governo federal intervir de forma efetiva para melhorar as práticas alimentares da população. Em primeiro lugar, para que haja intervenção governamental na alimentação da população é necessário que se conheçam as práticas alimentares atuais, as opiniões, bem como as variáveis socioeconômicas associadas. Cabe ao governo e aos profissionais de saúde uma intervenção, de forma a estimular o consumo adequado, utilizando os meios de comunicação que a população admite usar como fonte de informação sobre alimentação.

Em conclusão, a presente pesquisa permitiu verificar que os fatores sócio-demográficos, escolaridade, renda familiar e local de moradia estão associados aos padrões de consumo e às mudanças de comportamento alimentar, e identificar o perfil das práticas alimentares da população do Distrito Federal.

\section{Agradecimentos}

À CAPES (Coordenação de Aperfeiçoamento de Pessoal de Nível Superior) pelo apoio financeiro, na forma de bolsa de doutorado e ao PIBIC/CNPq (Programa Institucional de Bolsas de Iniciação Científica/Conselho Nacional de Desenvolvimento Científico e Tecnológico) pelas bolsas de Iniciação Científica para entrevistadores na realização das entrevistas domiciliares. 


\section{Referências}

1. Tomita LY, Cardoso MA. Avaliação da lista de alimentos e porções alimentares de questionário quantitativo de freqüência alimentar em população adulta. Cad Saúde Pública. 2002; 18: 1747-56.

2. Monteiro C, Mondini LE, Costa R. Mudanças na composição e adequação nutricional da dieta familiar nas áreas metropolitanas do Brasil (1988-1996). Rev Saúde Pública. 2000; 34: 251-8.

3. Freyre G. Casa-grande \& senzala. São Paulo: Record; 2000.

4. Cascudo LC. História da alimentação no Brasil. São Paulo: Global; 2004

5. IBGE (Fundação Instituto Brasileiro de Geografia e Estatística Brasileira). Estudo Nacional da Despesa Familiar - ENDEF: 1974-1975) - Tabela de composição de alimentos. Rio de Janeiro; 1977.

6. Brasil. Ministério da Saúde. INAN (Instituto Nacional de Alimentação e Nutrição). Pesquisa Nacional sobre Saúde e Nutrição: condições nutricionais da população brasileira: adultos e idosos. Brasília (DF); 1991.

7. Coitinho DC, Leão MM, Recine E, Sichieri R. Condições nutricionais da população brasileira: adultos e idosos. Brasília: Instituto Nacional de Alimentação e Nutrição; 1991.

8. IBGE (Fundação Instituto Brasileiro de Geografia e Estatística). Pesquisa sobre padrões de Vida - PPV: 19961997. Rio de Janeiro; 1998.

9. Sichieri R, Castro JFG, Moura AS. Fatores associados ao padrão de consumo alimentar da população brasileira urbana. Cad Saúde Pública. 2003;19: 47-53.

10. WHO (Word Health Organization). Obesity: preventing and managing the global epidemic. Report of a WHO Consultation on obesity. Geneva; 1998.

11. Instituto Danone. Alimentação equilibrada para a população brasileira. Florianópolis; 1998.

12. Simopoulos AP. The Mediterranean diets: what is so special about the diet of Greece? The scientific evidence. J Nutr. 2001; 13: 3065-73.

13. Sichieri R. Dietary patterns and their associations with obesity in the Brazilian city of Rio de Janeiro. Obes Res. 2002; 10: 43-49

14. Garcia RWD. Práticas e comportamento alimentar no meio urbano: um estudo no centro da cidade de São Paulo. Cad Saúde Pública. 1997; 13: 455-67.
15. Ades L, Kerbauy RR. Obesidade e indagações. Psicologia USP 2002; 13: 197-216.

16. Viebig RF, Valero MP. Desenvolvimento de um questionário de freqüência alimentar para o estudo de dieta e doenças não transmissíveis. Rev Saúde Pública. 2004; 38: 581-4.

17. Angelis RC. A. fome oculta: bases fisiológicas para reduzir seus riscos através de alimentação. São Paulo: Atheneu; 2000

18. Aquino RC, Philippi ST. Consumo infantil de alimentos industrializados e renda familiar na cidade de São Paulo. Rev Saúde Pública. 2002; 36: 655-60.

19. Nuzzo L. Avaliação do estado nutricional de adolescentes de uma instituição particular de ensino [dissertação de mestrado]. São Paulo: Faculdade de Saúde Pública da Universidade Federal de São Paulo; 1998.

20. Davies C, Claridge G, Fox J. Not just a pretty face: physical attractiveness and perfectionism in the eating disorders. J Eat Disord 2000; 27: 67-73.

21. Waterhouse D. Outsmarting the mother daughter food trap. Nova York: Hyperion; 2001

22. Poulain JP. Reflexões metodológicas para o estudo das práticas alimentares. Rev Nutr. 2003; 16: 365-86.

23. Garcia RWD. Representações sobre consumo alimentar e suas implicações em inquéritos alimentares: estudo qualitativo em sujeitos submetidos à prescrição dietética. Rev Nutr. 2004; 17: 15-28.

24. Mattos LL, Martins IS. Consumo de fibras alimentares em população adulta. Rev Saúde Pública. 2000; 34; 50-5.

25. Brasil. Ministério da Saúde. INAN (Instituto Nacional de Alimentação e Nutrição). Estudo multicêntrico de consumo alimentar. Brasília, DF; 1997.

26.IBGE (Fundação Instituto Brasileiro de Geografia e Estatística). Pesquisa de Orçamento Familiar: 1995-1996. Rio de Janeiro; 1997.

27. Garcia, RWD. A comida, a dieta, o gosto: mudanças na cultura alimentar urbana [tese de doutorado]. São Paulo: Instituto de Psicologia da Universidade de São Paulo; 1999.

28. Drapeau V, Després JP, Bouchar C, Allard L, Fournier G, Leblanc C, Tremblay A. Modifications in food-group consumption are related to long-term body-weight changes. Am J Clin Nutr. 2004; 80: 29-37.

Recebido em 3 de novembro de 2006

Versão final apresentada em 4 de abril de 2007

Aprovado em 16 de maio de 2007 